\title{
Cosmic-Ray Studies with Experimental Apparatus at LHC
}

\author{
Emma González Hernández ${ }^{1}$, Juan Carlos Arteaga ${ }^{2}$, Arturo Fernández Tellez ${ }^{1}$ and \\ Mario Rodríguez-Cahuantzi ${ }^{1, *(1)}$ \\ 1 Facultad de Ciencias Físico Matemáticas, Benemérita Universidad Autónoma de Puebla, Av. San Claudio y \\ 18 Sur, Edif. EMA3-231, Ciudad Universitaria, 72570 Puebla, Mexico; \\ emma.gonzalez.hernandez@cern.ch (E.G.H.); afernand@fcfm.buap.mx (A.F.T.) \\ 2 Instituto de Física y Matemáticas, Universidad Michoacana, 58040 Morelia, Mexico; arteaga@ifm.umich.mx \\ * Correspondence: mario.rodriguez@correo.buap.mx
}

Received: 11 September 2020; Accepted: 2 October 2020; Published: 15 October 2020

check for updates

\begin{abstract}
The study of cosmic rays with underground accelerator experiments started with the LEP detectors at CERN. ALEPH, DELPHI and L3 studied some properties of atmospheric muons such as their multiplicity and momentum. In recent years, an extension and improvement of such studies has been carried out by ALICE and CMS experiments. Along with the LHC high luminosity program some experimental setups have been proposed to increase the potential discovery of LHC. An example is the MAssive Timing Hodoscope for Ultra-Stable neutraL pArticles detector (MATHUSLA) designed for searching of Ultra Stable Neutral Particles, predicted by extensions of the Standard Model such as supersymmetric models, which is planned to be a surface detector placed 100 meters above ATLAS or CMS experiments. Hence, MATHUSLA can be suitable as a cosmic ray detector. In this manuscript the main results regarding cosmic ray studies with LHC experimental underground apparatus are summarized. The potential of future MATHUSLA proposal is also discussed.
\end{abstract}

Keywords: cosmic ray physics at CERN; atmospheric muons; trigger detectors; muon bundles

\section{Introduction}

Cosmic rays that reach Earth's atmosphere are made up of protons and heavy atomic nuclei. They arrive with energies between $10^{8} \mathrm{eV}$ and $10^{20} \mathrm{eV}$ [1-3]. It has been observed that cosmic rays are produced within our galaxy for energies less than $10^{18} \mathrm{eV}$ and beyond for energies greater than $10^{18} \mathrm{eV}$. However, the nature of cosmic rays at very high energies is not completely known, since its propagation through the outer space is influenced by galactic magnetic fields and thus the direction from which they arrive at the Earth is not the same as that of their source [3,4].

The composition, propagation and acceleration mechanisms of cosmic rays are not fully understood $[3,5,6]$. Thus, through the study of cosmic rays it is possible to deepen the knowledge of the collisions of the most energetic natural phenomena of the cosmos [7-9] with the Earth's atmosphere at the TeV energy scale [10-12]. For this reason cosmic rays are subject of an intense research around the world with different experimental techniques which allows to perform precise measurements on the primary cosmic ray composition, energy spectrum and arrival direction. Additional studies of neutrinos $[13,14]$ and gamma-rays $[7,15]$ are also desirable.

The characterization of primary cosmic rays depends on its energy: low energy primary cosmic rays can be studied with balloons or experimental setups placed in orbit equipped with excellent performance tracking and calorimeter systems, see, for example, CREAM [16,17] and AMS-02 [18,19] experimental programs. 
For cosmic rays with energies larger than $10^{14} \mathrm{eV}$, the Earth's atmosphere acts as a calorimeter $[1,3,20,21]$. The produced particles in the Extensive Air Showers (EAS) are detected at different altitude levels [21]. After the primary collision occurs, secondary particles are produced by a chain of secondary particle-physics reactions which dissipate the energy of the primary particle. Particle production continues until it reaches a maximum at a given energy threshold. After this point it decreases almost exponentially due to absorption processes in the atmosphere.

\section{Hadron Interaction Models}

The EAS has an electromagnetic (photons produced by the decay of $\pi^{0}$ and $\eta$ mesons), hadronic (nucleons) and leptonic components $\left(\mu^{ \pm}\right.$and $v_{\mu}, \bar{v}_{\mu}$ produced by the decay of $\pi^{ \pm}$and $K^{ \pm}$light mesons) [21,22]. Once the EAS starts its development, several hadron interactions occur from which there is a transfer of energy that feeds the electromagnetic and leptonic components of the EAS. Only about $10 \%$ of the charged particles in an EAS at high-energies and ground level are muons [23].

The hadronic interaction models are a key tool in Monte Carlo simulations which aims to describe the development of EAS. They have been updated accordingly to the evolution of collider experiments that produce results used for tuning such models. Current hadronic models, used for EAS simulations and some of them tuned with LHC data at seven TeV are: QGSJETII-03, QGSJETII-04, EPOS LHC, Sibyll 2.c (from CORSIKA V7.6300) and DPMJETIII.17-1 (references to each model can be found at [24]).

It has been discussed in [24] that approximately $70 \%$ of the uncertainties in the simulation of the depth of the shower maximum of EAS, $\left\langle X_{\max }\right\rangle$, comes from the first proton/nucleus-air interaction. The rest $30 \%$ is affected by the pion-air interactions. Moreover, $90 \%$ of the muon production is due to pion interactions and 10\% comes from the first interaction which can not be tested in a proper way for nuclear effects (nucleus-air interaction) with current hadronic interaction models. These nuclear effects tends to be the main source of a shift in $X_{\max }$ [24]. To reduce the known uncertainties in the description of the development of EAS using hadronic interactions models an improvement of both, pion-nucleus interactions and muon depth production, are desirable [24]. Thus, future models should take into account LHC data to make a significant improvement in the description of EAS. Another phenomena like heavy flavor production [25], creation of strong electromagnetic fields [26] and photo-production of vector mesons [27] can influence the measurements carried out by ground(under) apparatus. Therefore, it is important to perform as much as possible studies of the different components of the EAS to improve our understanding of the forward particle production at very high energies and consequently of the characteristics of the primary cosmic ray flux.

The number of muons $\left(N_{\mu}\right)$ produced through the development of the EAS is an observable sensitive to the characteristics of the primary cosmic ray. It is well known that $N_{\mu}$ has a linear dependence with the energy of the primary cosmic ray and with a power law with respect to its mass [28]. In this sense, several studies on the muon component of EAS have been carried out with experimental arrangements on the surface (Pierre Auger observatory [29], among others [30-32]) and underground (LEP [33] and LHC experiments at CERN [34,35]). The Pierre Auger Observatory reported a muon excess with respect to the theoretical expectations from hadronic interaction models for EAS with zenith angles larger than $67^{\circ}$ at energies for the primary cosmic rays of $10^{19} \mathrm{eV}$ [10]. Recently a direct measurement of the muon content in EAS for energies between $2 \times 10^{17}$ and $2 \times 10^{18} \mathrm{eV}$ was reported by Pierre Auger Observatory [36]. In this work the reconstructed muon density from data is larger than the predicted by theoretical models. Both results are intriguing and therefore have motivated several theoretical works that seek to give an interpretation of the Pierre Auger muon excess results. For example, it has been proposed a model based on the production of deconfined thermal fireballs which undergoes a sudden hadronization assuming a heavy component for the primary cosmic ray at ultra high energies [37]. Another scenario considers effects of string percolation in hadronic interactions [38]. This model has successfully described the data from RHIC for $A-A$ collisions with energies up to $200 \mathrm{GeV}$ and LHC for $p-p$ collisions at $7 \mathrm{TeV}$. Furthermore, 
models constructed under the assumption that the explanation of the muon excess reported by the Pierre Auger Observatory come from a reduction of the production or decay of $\pi^{0}$ due to a reduction of the transfer of energy from the hadronic part of the shower into the electromagnetic component of the EAS [39]. This is achieved through scenarios which considers physical phenomena like chiral symmetry restoration, pion decay suppression and pion production suppression [39]. In all these novel proposals, the physics observable of interest is the multiplicity of muons $\left(N_{\mu}\right)$ contained in the leptonic component of the EAS.

\section{Cosmic Ray Studies with LHC Experiments: ALICE and CMS}

Taking advantage of the tracking capabilities of the particle detectors designed for collider experiments at CERN it is possible to study the muon component, of the EAS, that reaches the underground apparatus. The experiments at LEP-CERN were the pioneers in the study of the muon component of EAS using dedicated collider underground detectors. The ALEPH [40] and DELPHI [41] collaborations reported the observation of high multiplicity atmospheric muon events that could not be explained using the hadronic interaction models available at that time. The main conclusion of LEP experiments about these phenomena was that events with intermediate multiplicity of muons $\left(N_{\mu} \leq 50\right)$ are reproducible using the standard particle production mechanisms, but the high multiplicity muon events $\left(N_{\mu}>100\right)$ occur with a frequency at least two orders of magnitude higher than the predictions of standard hadronic interaction models [33]; even with the assumption of a heavy component in the primary cosmic ray.

In recent years, LHC underground experimental apparatus, ALICE [35] and CMS [34], have studied muon bundles events and single cosmic charge ratio, $R_{\mu}$, respectively. $R_{\mu}$ can be used to improve the understanding of the mechanism of multiple production of pions and kaons in the atmosphere as the EAS develops. It is also useful to improve the description of forward events with hadronic interactions models and thus constraining theoretical predictions at ultra-high energies. $R_{\mu}$ provides information about the forward production of the number of $\pi^{+}$and $K^{+}$over $\pi^{-}$and $K^{-}$ respectively. A nice review about the importance of $R_{\mu}$ measurement can be found in [42-44].

Prior to the LHC commissioning in 2009, the CMS Collaboration collected around 295 millions of cosmic ray events. With this sample they measured $R_{\mu}$ in a wide range of momenta. The result reported by CMS in [45], $R_{\mu}=1.2766 \pm 0.0032$ (stat.) \pm 0.0032 (syst.), is in excellent agreement with previous measurements. $\mathrm{Up}$ to now, the CMS results are the most precise measurement of $R_{\mu}$ for a momentum less than $100 \mathrm{GeV} / c$.

In the future, it will worth to perform a study of the cosmic charge ratio with respect to the muon multiplicity. This would enable us to discriminate muons coming from a heavy component of the primary cosmic ray. At very high energies, the heavy flavour component of the the primary cosmic ray and the EAS may be significant. Moreover, while for single muon events $R_{\mu}=1.28$ means a larger abundance of a light cosmic ray component over heavier elements, it may be that for multi-muon events this effect is diminished [46].

\section{Study of Muon-Bundles with ALICE}

ALICE is one of the main detectors at LHC. It is devoted to the study of the matter created in lead-lead collisions [35]. Located 52 meters underground, this experiment has been used as a cosmic ray detector to study muons with an energy threshold of $16 \mathrm{GeV}$. The tracking system of ALICE allows the reconstruction of charge and momentum of the muons that cross its central region. With this information, the ALICE cosmic ray group studied the rate of events with more than 100 atmospheric muons reconstructed by the ALICE's Time Projection Chamber (TPC). They found that the rate of such events can be described using the QGSJET-II-04 [47] model (tuned with TOTEM-LHC data [48]) assuming a heavy component (Fe) of the primary cosmic ray with energies greater than than $10^{16} \mathrm{eV}$ [49]. Furthermore, the muon multiplicity distribution reconstructed with the ALICE cosmic data is similar to the one reported by ALEPH and DELPHI experiments [33], see Figure 1, where five 
events with more than 100 atmospheric muons are seen. This is the first time that the rate of high muon multiplicity (HMM) events was described using the available hadronic interaction models. As can be seen in Table 1, the uncertainty of ALICE data is large. For this reason, it will be important to confirm the ALICE result with new data to make an additional comparison with revised hadronic interaction models [42].

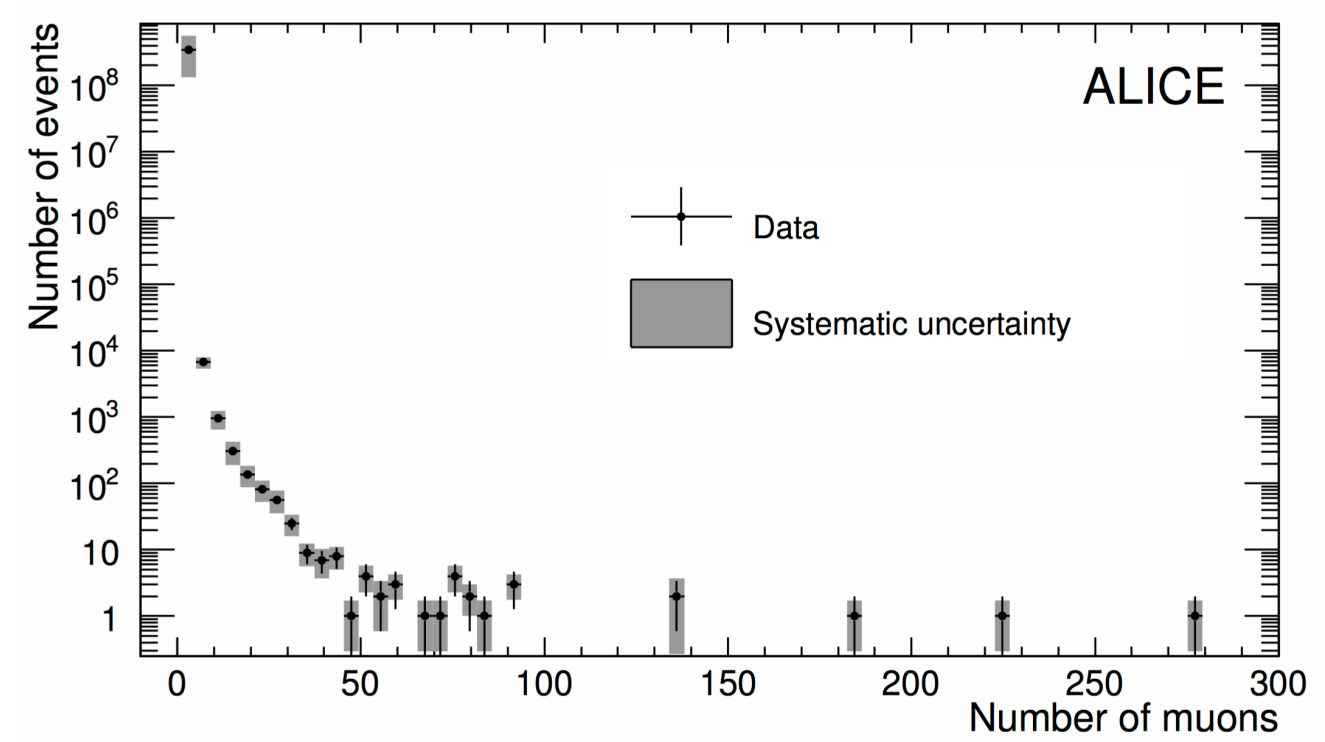

Figure 1. Atmospheric muon multiplicity distribution reported by the ALICE Collaboration. Figure taken from [49].

Table 1. Summary of ALICE results on frequency of high muon multiplicity events.

\begin{tabular}{cccccc}
\hline \multirow{2}{*}{ HMM Events } & \multicolumn{2}{c}{ CORSIKA 6990 } & \multicolumn{2}{c}{ CORSIKA 7350 } & \\
\cline { 2 - 6 } & \multicolumn{2}{c}{ QGSJET II-03 } & QGSJET II-04 & Data \\
\cline { 2 - 6 } & Proton & Iron & Proton & Iron & \\
\hline Period [days per event] & 15.5 & 8.6 & 11.6 & 6.0 & 6.2 \\
Rate [ $\left.\times 10^{-6} \mathrm{~Hz}\right]$ & 0.8 & 1.3 & 1.0 & 1.9 & 1.9 \\
Uncertainty $(\%)$ (syst + stat) & 13 & 16 & 8 & 20 & 49 \\
\hline
\end{tabular}

A comparison of the ALICE data with the hadronic interaction models, QGSJET-II-03/04 [47] available in CORSIKA [50] was also carried out by ALICE. Figure 2 shows that the ALICE data points are between proton and Fe. At low multiplicities, ALICE's data points are close to the proton estimation. As the multiplicity of muons increases, the experimental data points get closer to the Fe Monte Carlo points.

Some theoretical proposals suggest that the events reported by ALICE are produced by strangelets, hypothetical nuclear matter composed of doublets or triplets of strange quarks, with possible extra-galactic origin [51]. In this scenario, the muon bundles detected by ALICE are understood to be created by a mixture of strange quark matter. This matter loses mass when penetrates in the atmosphere. In Figure 3, the Monte Carlo prediction for SQM is shown. Such model describes the rate of the high muon multiplicity events seen by ALICE by allowing for a relatively small admixture of strangelets of the same total energy [51]. 


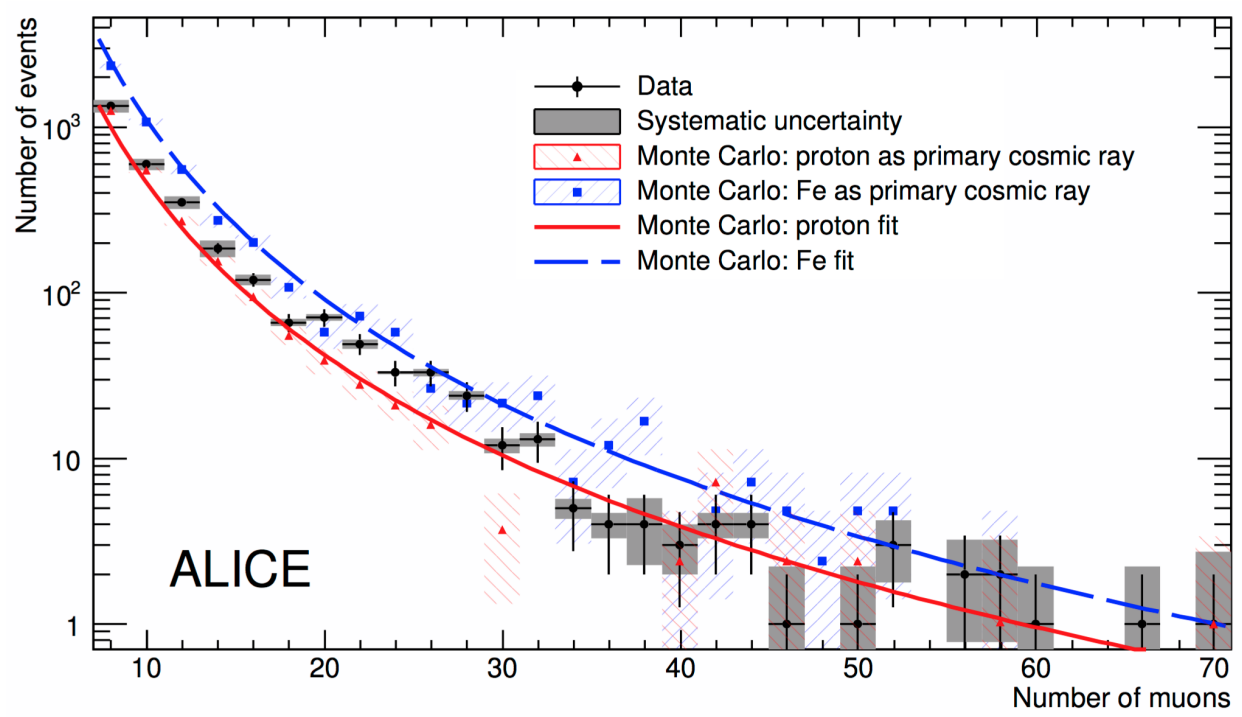

Figure 2. Atmospheric muon multiplicity distribution reported by ALICE. Figure taken from [49].

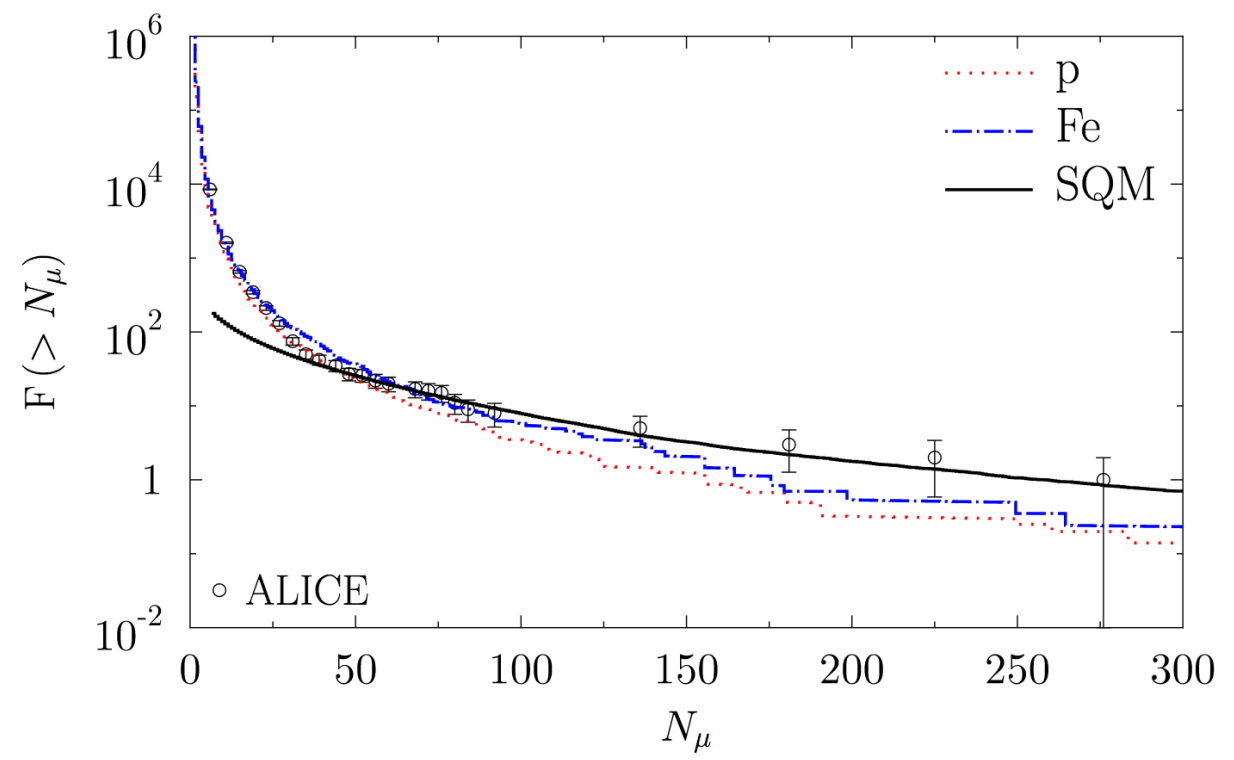

Figure 3. The SQM model adjustment to ALICE data is shown (black line) figure taken from [51].

Other proposal is that the HMM events observed by ALICE are indicative of the creation of the quark gluon plasma due to the interaction of primary cosmic rays with the Earth's atmosphere, where the primary cosmic ray is made of a heavy nuclei with a very high energy that it is not possible to access in colliding experiments in laboratories [52].

\section{Future Experimental Arrangement: MATHUSLA Proposal}

The LHC experiments, and the accelerator itself, are currently planning major upgrades for their operation during the scheduled high luminosity run of the LHC. Taking advantage of the long technical stop of the LHC for such upgrade, a surface array detector is being proposed to be installed at the surface above the ATLAS [53] and/or CMS [34] experiment(s) (see Figure 4). This new detector, known as MATHUSLA [54], is being designed for the search of neutral long-lived particles (LLPs) that might be produced, with a displaced vertex, in p-p collisions during the LHC high luminosity run. The observation of LLPs would imply the discovery of physical phenomena beyond the Standard Model, specially of those models based in supersymmetry (SUSY) [55]. The MATHUSLA detector will 
have a sensitive detection area of $100 \times 100 \mathrm{~m}^{2}$ at ground level at an altitude where the atmospheric depth is $\sim 1000 \mathrm{~g} / \mathrm{cm}^{2}$. The design of MATHUSLA is modular. It will consist of 100 individual $9 \mathrm{~m} \times 9 \mathrm{~m}$ modules placed in a rectangular surface above the ATLAS or CMS location at LHC Points 1 or 5, respectively. Each individual MATHUSLA module will have 9 detector planes constructed with plastic scintillator pieces and wavelength shifter bars and SiPM photo sensors: 2 at the bottom, 5 at the top separated by 1 meter each other and 5 meters from 2 tracking layers (see Figure 5). An additional RPC top tracking layer is under consideration. Thus MATHUSLA is expected to have an excellent efficiency for the detection of cosmic ray events with energies between $10^{15}$ and $10^{17} \mathrm{eV}$.

Up to date, the MATHUSLA project is under discussion of LHCC committee. It is expected that MATHUSLA collaboration will release a technical design report by the end of 2021. For the coming years the construction and commissioning of a prototype is planned. This prototype will be located somewhere at LHC Point 5 near the interaction point of CMS detector. The MATHUSLA group will explore different technologies for data acquisition and detector development. Furthermore, they are planning to perform several simulation studies of LLPs decay and cosmic ray events. The MATHUSLA group is open to new members. An update of the MATHUSLA project can be found in [56].

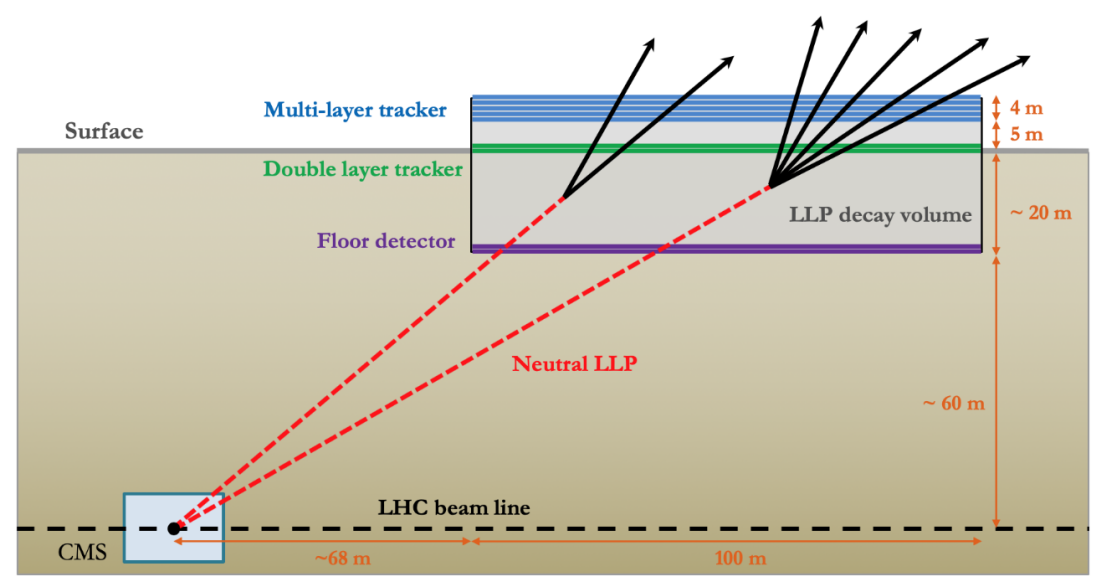

Figure 4. MATHUSLA expected location above CMS [56].
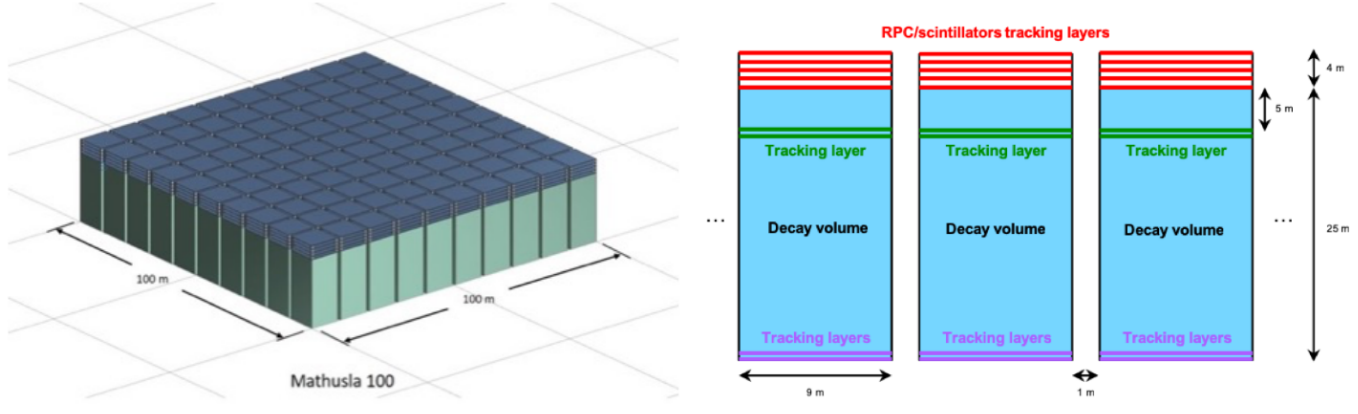

Figure 5. Concept of MATHUSLA detector [56].

As a cosmic ray detector, MATHUSLA will sample, at observation level, the front of the air showers that the cosmic rays produce in the atmosphere. Measurements are expected to be performed event-by-event with the MATHUSLA tracking system alone or in combination with the CMS/ATLAS 
underground detector in what will be called the standalone (STD) and combined (CB) modes, respectively. Some shower observables in each case are listed below:

1. STD: It will provide data about the arrival times, individual trajectories and spatial distributions of the charged particles produced along the EAS front at observation level. With this information, MATHUSLA will be able to reconstruct the location of the shower core, the direction of the shower axis, the slope of the radial distribution of particle densities (also known as shower age, $s$ ) and the shower size of the EAS. The shower size reveals the energy of the primary cosmic ray, while the shower age is sensitive to the mass of the primary cosmic ray.

2. CB: If MATHUSLA triggers the underground CMS/ATLAS detector in order to measure simultaneously the passage of shower events, then additional EAS information on high energy muons ( $E_{\mu}^{t h} \gtrsim 50 \mathrm{GeV}-70 \mathrm{GeV}$ for vertical incidence) will be available. Using the capabilities of CMS/ATLAS shower observables like the local number of muons per $\mathrm{m}^{2}$, the $p_{\mu}$ distributions and the $\mu^{+} / \mu^{-}$ratio per shower event will be also measured. The combined data from the surface and underground detectors about EAS events could improve our analyses of composition of cosmic rays. Besides, coincident measurements of MATHUSLA with the CMS/ATLAS will allow to study the phenomenon of muon bundles in connection with extensive air showers.

\subsection{Stand Alone Mode}

In STD mode, it is expected that the MATHUSLA tracking system will provide information about the arrival times, the number of charged particles, $N_{c h}$, and their spatial distributions at observation level as well as their individual trajectories. These measurements will allow the reconstruction of the shower size, the arrival direction of the primary cosmic ray, the lateral shower age, $s$, and the shower core of the EAS event. The charged component here is considered as the sum of $e^{ \pm}$and $\mu^{ \pm}$shower particles. For vertical EAS, $N_{c h}$ is dominated by the electron component, while for inclined showers, $N_{c h}$ contains mostly muons. The charged hadron component is not considered, as it is not dominant.

In general, for EAS detectors that make use of the sampling technique, the core of the shower is obtained by looking for the region of larger particle density, while the arrival direction of the shower can be determined from the arrival time information of the particles in the shower front. In this case, at first order of approximation, the EAS front is fitted to a plane and the direction of the shower is obtained from the normal vector to the surface. A rough estimation indicates that a plane shower front which travels to the velocity of light, $c$, takes a time of around $\Delta t=d \cos \theta / c$ to cross the detector, where $d$ is the diameter of the array and $\theta$ is the arrival zenith angle of the EAS [57].

The total number of produced particles $(N)$ in the EAS is estimated by fitting the measured density map of particles to a proper function $\rho(r)$ which depends on the radial distance, $r$, to the core. This fitted density function is then integrated over $r$, thus giving an estimation of the total number of shower particles [57],

$$
N=2 \pi \int_{0}^{\infty} r \rho(r) d r
$$

The steepness of the density map gives a measurement of the degree of development in the atmosphere of the EAS. The former is parameterized in the density function used for the fit of $N$ through a quantity denoted by $s$ and called the shower age and it is obtained from the best fit to the measured density profile. An example of a lateral density profile is the NKG function [58] derived for the context of electromagnetic EAS:

$$
\rho(r)=N \frac{C(s)}{r_{0}^{2}}\left(\frac{r}{r_{0}}\right)^{s-2}\left(1+\frac{r}{r_{0}}\right)^{s-4.5}
$$

where $C(s)$ is a normalization factor and $r_{0}$ is the Moliere radius, which measures the degree of spread of low energy electrons in the EAS. 
Once the observables of the EAS are obtained, the main properties of the primary cosmic ray may be estimated. For example, the arrival direction of the primary particle will correspond to the direction of the axis of the shower, while the cosmic ray energy can be estimated using the total number of charged particles using proper calibrated formulas derived from Monte Carlo simulations. To investigate the nature of cosmic rays, the lateral shower age might be explored, as it depends on the composition of the primary particle $[59,60]$. However, complementary measurements with other observables like the muon content of EAS might be necessary.

\subsection{Combined Mode}

Working in tandem with the underground ATLAS or CMS detector, simultaneous measurements of the EAS might be performed during the LHC run time. For these events the underground detector would provide local data of high energy muons ( $E_{\mu}^{\text {th }} \gtrsim 50 \mathrm{GeV}-70 \mathrm{GeV}$ for vertical incidence) at the shower. Using the capabilities of the underground detectors shower observables like the number of muons per $\mathrm{m}^{2}$, energy-momentum distributions, zenith angle distributions and very precise charge identification $\left(\mu^{+}, \mu^{-}\right)$might be also measured. Additionally, coincident measurements of MATHUSLA with the CMS/ATLAS will allow to study the phenomenon of muon bundles in connection with EAS.

\subsection{Comparison with Other EAS Detectors}

In the energy range around $\mathrm{PeV}^{\prime} \mathrm{s}$, there are now other detectors taking measurements of cosmic rays at different locations and altitudes, with different areas and techniques. In Table 2, a compilation of some past/present/future EAS PeV detectors is presented and compared with MATHUSLA.

Table 2. Experiments whose data could be competitive with results of MATHUSLA. Sci. means scintillators, FD means fluorescence detectors, WCD, water cherenkov detectors, ICD, ice cherenkov detectors, TD, particle tracking detector, CD, calorimeter, IACT, Imaging Air Cherenkov telescope, RPC, Resistive Plate Chambers, and AC, Air Cherenkov. From the list on the table ARGO-YBJ and KASCADE are no longer active. LHASSO is under construction.

\begin{tabular}{|c|c|c|c|c|}
\hline Experiment & $\begin{array}{c}\text { Energy Range } \\
\quad(\mathrm{PeV})\end{array}$ & $\begin{array}{l}\text { Altitude } \\
\text { (m a. s. 1) }\end{array}$ & $\begin{array}{c}\text { Size } \\
\left(10^{4} m^{2}\right)\end{array}$ & Technique \\
\hline MATHUSLA & $(1,32)$ & $380-436$ & 1 & RPC, TD \\
\hline ARGO-YBJ [61] & $(0.1,3)$ & 4300 & 0.7 & RPC, TD \\
\hline KASCADE [62] & $\left(1,10^{2}\right)$ & 110 & 4 & Sci, TD, CD \\
\hline HAWC-Outrigger [63] & $\left(10^{-4}, O(1)\right)$ & 4100 & 11 & WCD \\
\hline Taiga [64] & $>0.1$ & 675 & 25 & IACTs \\
\hline IceTop [65] & $\left(1,10^{3}\right)$ & 2835 & 100 & ICD \\
\hline LHAASO $[66,67]$ & $\left(10^{-4}, 10^{2}\right)$ & 4410 & 100 & WCD, AC, Sci. \\
\hline TALE (TA) [66] & $\left(30,10^{4}\right)$ & 1550 & $10^{3}$ & FD, Sci. \\
\hline
\end{tabular}

Compared with these air shower detection systems, MATHUSLA will have several advantages. For example:

- Full coverage with robust tracking: That is an advantage from the usage of different layers of fine segmented RPCs, which no other EAS ground-based detector posses. Full coverage was achieved by the ARGO-YBJ detector [61], but since it counted with a single layer of RPCs, it did not count with the tracking capabilities that are expected for MATHUSLA. Other detectors like KASCADE [62] counted with muon tracking $\left(150 \mathrm{~m}^{2}\right)$ and calorimeter $\left(16 \times 20 \mathrm{~m}^{2}\right)$ systems but the coverage was reduced to less than $1 \%$. Besides their area, compared with MATHUSLA, was reduced in size.

- Highly granular measurements of the shower's temporal and spatial structure: From the list of PeV EAS detectors provided, only ARGO-YBJ [61] and the muon tracking and calorimeter systems of KASCADE [62] were able to perform fine measurements of the temporal/spatial structure of 
the showers for the charged component of EAS in the former case, and the muon and hadron components in the latter.

- Tandem measurements with a fine muon underground detector: Underground muon tracking detectors have been used before in other air shower facilities, for example, at the KASCADE observatory [62], however they were lacking the capabilities of the ATLAS/CMS detectors, which are also able to measure the charge and spectrum of individual muons. The capabilities of MATHUSLA might be further expanded when working in coincidence mode with the CMS or ATLAS detector. That will complement the EAS measurements of MATHUSLA with detailed information of the muon content at high energies, which will be useful for the study of the spectrum and composition of cosmic rays, and the understanding of high-energy hadronic interactions in the forward region, where there are significant uncertainty calculations. In addition studies of muon bundles might be devised in connection with air showers.

MATHUSLA working as a cosmic ray hodoscope will have several advantages over other air shower arrays regarding the measurements of EAS properties. Its special design, which considers several detector planes with RPCs of big size, will allow on the one side to enhance its angular resolution for air showers. For a surface EAS detector system, the angular precision is determined from the area of the array, the corresponding spacing, $d[68]$. It depends roughly of $1 / d$. Hence, it is expected a better angular resolution for MATHUSLA than for other EAS detector systems. This resolution could be even further improved using also the information of the tracking system of MATHUSLA.

On the other hand, the fine segmentation and full coverage of MATHUSLA will allow to study in detail the shape of the lateral density distribution of EAS at the shower front as carried out at the ARGO-YBJ experiment [60]. That will increase the precision of MATHUSLA in the location of shower cores and it will allow a better determination of both the total number of charged particles $\left(N_{c h}\right)$ and the lateral shower age $s$ of the events, as there will be more points available for the fit of the density maps at ground level from which $N_{c h}$ and $s$ are obtained. Increasing the resolution of the location of the shower core will improve the determination of the arrival direction of the EAS and of $N_{c h}$ and $s$. A better calculation of $N_{c h}$ will permit a better estimation of the primary energy of the event. However, the disadvantage of MATHUSLA will be that the energy and composition studies based on $N_{c h}$ and $s$ will rely on simulations from hadronic interaction models. Here, calorimetric measurements of the total energy of the EAS in the atmosphere using cherenkov and fluorescence telescopes $[64,66]$ or radio techniques give more reliable results. KASCADE had an additional advantage over MATHUSLA regarding the energy calibration, and it is that it used two different shower observables for the shower size, $N_{c h}$ and $N_{\mu}$ (number of muons), which reduce the composition uncertainties in the energy estimation of the primary particle.

Cherenkov and fluorescence telescopes, however, have a small duty cycle, as they can monitor the sky only during moon-less nights. The advantage of the surface EAS detector systems as MATHUSLA is that they can monitor the sky day and night, independently of the weather conditions, increasing the statistics in comparison with arrays where Cherenkov/fluorescence telescopes are used.

A further advantage is that there will be several fine time/spatial measurements at MATHUSLA that will also allow to study the physics of air showers and use them to test the hadronic interaction models commonly used to calibrate the cosmic ray measurements in EAS experiments. In Table 3, we summarize some advantages of MATHUSLA and compare them with those of other air shower arrays.

It is important to note that experimental arrays, such as LHAASO detectors [67], have been designed to study with high accuracy gamma and cosmic ray events in a wider energy range, from $10^{11} \mathrm{eV}$ to $10^{17} \mathrm{eV}$. LHAASO will be an excellent instrument to study the cosmic ray anisotropy around the knee and it is expected to be sensitive to the masses of the primary cosmic ray. LHAASO will be a hybrid detector that combines plastic scintillator detectors and under/ground water Cherenkov tanks. Thus, LHAASO will be able to detect and to study with high efficiency EAS with energies between $10^{11} \mathrm{eV}$ and $10^{17} \mathrm{eV}$, a wider range than MATHUSLA with an extension $76 \%$ larger than MATHUSLA. 
From the above, MATHUSLA is not expected to be competitive with experiments such as LHAASO. MATHUSLA will be a new category of detector, with performance that might be located between that for EAS detectors with Cherenkov telescopes and surface arrays with particle detectors on the basis of the precision that it might be achieved in aspects such as the reconstruction of the energy of the EAS. However, its performance will go beyond the above instruments with regard to the spatial-temporal sampling of the shower front, which might improve also the reconstruction of the arrival direction and core position of EAS in comparison with that from the above detector systems. Therefore, MATHUSLA will provide a brand new way of observing the cosmic ray sky and study the EAS phenomena at PeV energies.

Table 3. Comparative table of characteristic EAS measurements for experiments whose data could be competitive with results of MATHUSLA. Full coverage here is referred as the ratio of the sum of physical areas of all the detectors in each array with respect to the covered area of the array. From the list on the table ARGO-YBJ and KASCADE are no longer active.

\begin{tabular}{cccccc}
\hline Observatory & Full & Spatial & Angular & Energy & CR Composition \\
\hline Observatory & Coverage & Resolution & Resolution & Precision & Capabilities \\
\hline MATHUSLA & $100 \%$ & Very good & Very good & Good & Limited by statistics \\
ARGO-YBJ [61] & $93 \%$ & Very good & Good & Good & Good \\
KASCADE [62] & $<2 \%$ & Good & Good & Good & Very good \\
HAWC-Outrigger [63] & $0.8-62 \%$ & Good & Good & Good & In investigation \\
IceTop [65] & $0.044 \%$ & Good & Good & Good & In investigation \\
TALE (TA) [66,67] & O(1\%) & Good & Good & Very good & In investigation \\
\hline
\end{tabular}

\section{Conclusions}

Underground experiments at CERN have provided results in field of cosmic ray physics. LEP results on the multi-muon analysis helped to test hadronic interaction models which could not describe the LEP data. Moreover, ALICE and CMS experiments at LHC used their cosmic data to perform analysis on multi-muon events and single cosmic charge ratio respectively. The CMS measurement is in perfect agreement with the previous results given by underground experiments. Future measurements of the dependence of this ratio on the muon multiplicity will help to achieve a deeper understanding of the hadronic interactions at very high energies.

The ALICE Collaboration at CERN started a dedicated cosmic ray physics program to study muon bundles with an underground apparatus. During the Run I data taking period of the LHC, ALICE collected 30.8 effective days of cosmic ray data. With this sample, they were able to measure the multiplicity of atmospheric muons produced in EAS detecting the same type of high muon multiplicity events as ALEPH and DELPHI experiments. Particularly ALICE observed 5 HMM events $\left(N_{\mu}>100\right)$. In the ALICE cosmic ray physics paper [49] is shown that the frequency of such kind of events can be reproduced in terms of the QGSJET II-04 model. Moreover, the ALICE cosmic ray group found that these events are produced in EAS from primary cosmic rays whose composition is dominated by a heavy component ( $\mathrm{Fe}$ ) with energies larger than $10^{16} \mathrm{eV}$. They also determined the cores of the observed showers were located very close to the ALICE detector and that they had zenith angles less than $50^{\circ}$. This result places significant constraints on alternative, more exotic production mechanisms within its uncertainties (systematic + statistical) of about $49 \%$. This measurement may be improved with the new data collected by ALICE between 2015 and 2018 equivalent to about 60 effective days of cosmic data.

In the near future horizon, a detector with the characteristics of MATHUSLA can provide valuable information to extend EAS studies. The size of MATHUSLA might not allow to separate all the different mass groups of cosmic rays as in [69] around the knee due to the available statistics, but it 
could help to separate the light and heavy mass groups of primary cosmic rays allowing to check the observations of KASCADE.

MATHUSLA will be a new class of EAS detector for the study of cosmic rays at PeV energies. It could provide quality data on air showers induced by cosmic rays with better resolution than other past and present experiments. In particular, on the shower arrival direction, EAS core position, the density and time distributions as well as the total number of charged particles in shower events with energies around the knee, in standalone mode, without assuming an $e / \mu$ discrimination system. In combined detection mode, it will take simultaneously data together with the CMS or ATLAS detectors. The underground detectors will provide complementary measurements of the EAS, particularly, about the muon content at energies above $50 \mathrm{GeV}$ to $70 \mathrm{GeV}$ for vertical incidence. In STD mode, MATHUSLA could be used to measure the all-particle energy spectrum, study the cosmic ray anisotropies, study the muon content of inclined events, tests hadronic interaction models and possibly to perform composition studies of cosmic rays using the age parameter for the charged component of EAS. In CB mode, it will extend the number of test of hadronic interactions and it will permit to study other EAS phenomena as muon bundles in connection with air shower events. If $e / \mu$ is implemented in MATHUSLA, the studies on the energy spectrum and composition of cosmic rays will be strongly improved. However, even without an $e / \mu$ discrimination a wide cosmic ray physics case could be expected. At the date, the MATHUSLA project is under consideration of the LHC scientific committee. It is open to new members.

Author Contributions: Conceptualization, M.R.-C. and E.G.H; methodology, M.R.C. and E.G.H.; investigation, M.R.-C., E.G.H. and J.C.A.; formal analysis, M.R.-C., E.G.H. and J.C.A.; investigation, M.R.-C., E.G.H. and J.C.A.; resources, M.R.-C. and A.F.T.; writing-original draft preparation, M.R.-C.; writing-review and editing, M.R.-C. and E.G.H.; supervision, M.R.-C.; project administration, M.R.-C.; funding acquisition, M.R.-C. All authors have read and agreed to the published version of the manuscript.

Funding: This research was funded by Consejo Nacional de Ciencia y Tecnología, México (CONACyT) grant numbers A1-S-13525 and A1-S-16215. The authors thankfully acknowledges the permission to use computer resources, the technical advise and the support provided by Laboratorio Nacional de Supercómputo del Sureste de México (LNS), a member of the CONACyT national network of laboratories, with resources from grant number 201701035C.

Conflicts of Interest: The authors declare no conflict of interest.

\section{References}

1. Bluemer, J.; Engel, R.; Hoerandel, J.R. Cosmic Rays from the Knee to the Highest Energies. Prog. Part. Nucl. Phys. 2009, 63, 293-338 [CrossRef]

2. Grenier, I.A.; Black, J.H.; Strong, A.W. The Nine Lives of Cosmic Rays in Galaxies. Annu. Rev. Astron. Astrophys. 2015, 53, 199-246. [CrossRef]

3. Mollerach, S.; Roulet, E. Progress in high-energy cosmic ray physics. Prog. Part. Nucl. Phys. 2018, 98, 85-118. [CrossRef]

4. The Pierre Auger Collaboration. Observation of a large-scale anisotropy in the arrival directions of cosmic rays above $8 \times 10^{18} \mathrm{eV}$. Science 2017, 357, 1266-1270. [CrossRef]

5. Giacinti, G.; Kachelrie, M.; Semikoz, D.V. Escape model for Galactic cosmic rays and an early extragalactic transition. Phys. Rev. D 2015, 91, 083009. [CrossRef]

6. Gaisser, T.K.; Stanev, T.; Tilav, S. Cosmic ray energy spectrum from measurements of air showers. Front. Phys. 2013, 8, 748-758. [CrossRef]

7. Fermi-LAT Collaboration. Detection of the Characteristic Pion-Decay Signature in Supernova Remnants. Science 2013, 339, 807-811. [CrossRef] [PubMed]

8. HESS Collaboration. Acceleration of petaelectronvolt protons in the Galactic Centre. Nature 2016, 531, 476-479. [CrossRef]

9. Fang, K.; Murase, K. Linking high-energy cosmic particles by black-hole jets embedded in large-scale structures. Nat. Phys. 2018, 14, 396-398. [CrossRef]

10. The Pierre Auger Collaboration. Muons in air showers at the Pierre Auger Observatory: Mean number in highly inclined events. Phys. Rev. D 2019, 91, 032003. 
11. The Pierre Auger Collaboration. Testing Hadronic Interactions at Ultrahigh Energies with Air Showers Measured by the Pierre Auger Observatory. Phys. Rev. Lett. 2016, 117, 192001. [CrossRef] [PubMed]

12. The Pierre Auger Collaboration. Inferences on Mass Composition and Tests of Hadronic Interactions from 0.3 to $100 \mathrm{EeV}$ using the water-Cherenkov Detectors of the Pierre Auger Observatory. Phys.Rev. D 2017, 96, 122003. [CrossRef]

13. ICECUBE Collaboration. Neutrino emission from the direction of the blazar TXS 0506+056 prior to the IceCube-170922A alert. Science 2018, 361, 147-151. [CrossRef] [PubMed]

14. Becker, J.K. High-energy neutrinos in the context of multimessenger physics. Phys. Rep. 2008, 458, $173-246$. [CrossRef]

15. Knoedlseder, J. The future of gamma-ray astronomy. Comptes Rendus Phys. 2016, 17, 663-678. [CrossRef]

16. CREAM Collaboration. The Cosmic Ray Energetics And Mass (CREAM) instrument. Nucl. Instrum. Methods A 2007, 579, 1034-1053. [CrossRef]

17. CREAM Collaboration. Discrepant hardening observed in cosmic-ray elemental spectra. Astrophys. J. 2010, 714, L89-L93. [CrossRef]

18. AMS Collaboration. The Alpha Magnetic Spectrometer on the International Space Station. Int. J. Mod. Phys. E 2012, 21, 1230005. [CrossRef]

19. AMS Collaboration; Aguilar, M. Precision Measurement of the Proton Flux in Primary Cosmic Rays from Rigidity $1 \mathrm{GV}$ to $1.8 \mathrm{TV}$ with the Alpha Magnetic Spectrometer on the International Space Station. Phys. Rev. Lett. 2015, 114, 171103. [CrossRef]

20. Kampert, K.; Watson, A.A. Extensive air showers and ultra high-energy cosmic rays: a historical review. Eur. Phys. J. 2012, H37, 359-412. [CrossRef]

21. Engel, R.; Heck, D.; Pierog, T. Extensive Air Showers and Hadronic Interactions at High Energy. Ann. Rev. Nucl. Part. Sci. 2011, 61, 467-489. [CrossRef]

22. Gaisser, T.; Engel, R.; Resconi, E. Cosmic Ray and Particle Physics; Cambridge University Press: Cambridge, UK, 1990.

23. Grupen, C. Astroparticle Physics; Springer: New York, NY, USA, 2005.

24. Pierog, T. Air Shower Simulations with a New Generation of post-LHC Hadronic Interaction Models in CORSIKA. In Proceedings of the 35th International Cosmic Ray Conference, Busan, Korea, 10-20 July 2017.

25. Goncalvez, V.; Machado, T. Saturation Physics in Ultra High Energy Cosmic Rays: Heavy Quark Production. J. High Energy Phys. 2007, 4, 28. [CrossRef]

26. Ayala, A.; Castaño-Yepes, J.D.; Dominguez, C.A. ; Hernandez, L.A.; Hernandez-Ortiz, S.; Tejeda-Yeomans, M.E. Prompt photon yield and elliptic flow from gluon fusion induced by magnetic fields in relativistic heavy-ion collision. Phys. Rev. D 2017, 96, 014023; Erratum in Phys. Rev. D 2017, 96, 119901. [CrossRef]

27. ALICE Collaboration. Coherent $J / \psi$ photoproduction in ultra-peripheral $\mathrm{Pb}-\mathrm{Pb}$ collisions at $\sqrt{s_{N N}}=2.76$ TeV. Phys. Lett. B 2013, 718, 1273-1283. [CrossRef]

28. Matthews, J. A Heitler model of extensive air showers. Astropart. Phys. 2005, 22, 387-397. [CrossRef]

29. Auger Collaboration. Properties and performance of the prototype instrument for the Pierre Auger Observatory. Nucl. Inst. Meth. Phys. A 2004, 523, 50-95. [CrossRef]

30. Tunka Collaboration. Tunka-133: Results of 3 year operation. Nucl. Inst. Meth. Phys. A 2014, 756, $94-101$. [CrossRef]

31. TALE Collaboration. The Cosmic-Ray Energy Spectrum between $2 \mathrm{PeV}$ and $2 \mathrm{EeV}$ Observed with the TALE detector in monocular mode. Astrophys. J. 2018, 865, 1-74.

32. KASKADE Collaboration. The cosmic-ray experiment KASKADE. Nucl. Inst. Meth. Phys. A 2003, 513, 490-510. [CrossRef]

33. Travnicek, P. Detection of cosmic ray events by LEP experiments. Czech. J. Phys. 2006, 56, A185-A200. [CrossRef]

34. CMS Collaboration. The CMS experiment at the CERN-LHC. Jinst 2008, 3, S08004.

35. ALICE Collaboration. The ALICE experiment at the CERN LHC. Jinst 2008, 3, S08002.

36. The Pierre Auger Collaboration. Direct measurement of the muonic content of extensive air showers between $2 \times 10^{17}$ and $2 \times 10^{18} \mathrm{eV}$ at the Pierre Auger Observatory. Eur. Phys. J. C 2020, 80, 751. [CrossRef]

37. Anchordoqui, L.A.; Goldberg, H.; Weiler, T.J. Strange fireball as an explanation of the muon excess in Auger data. Phys. Rev. D 2017, 95, 063005. [CrossRef] 
38. Alvarez-Muniz, J.; Cazon, L.; Conceicao, R.; de Deus, J.D.; Pajares, C.; Pimenta, M. Muon production and string percolation effects in cosmic rays atthe highest energies. arXiv 2012, arXiv:1209.6474.

39. Allen, J.D.; Farrar, G.R. Testing models of new physics with UHE air shower observations. In Proceedings of the 33rd ICRC, Rio de Janeiro, Brazil, 9-12 December 2019.

40. Avati, V.; Dick, L.; Eggert, K.; Ström, J.; Wachsmuth, H.; Schmeling, S.; Ziegler, T.; Brühl, A.; Grupen, C. Cosmic multi-muon events observed in the underground CERN-LEP tunnel with the ALEPH experiment. Astropart. Phys. 2003, 19, 513-523. [CrossRef]

41. DELPHI Collaboration. Study of multi-muon bundles in cosmic ray showers detected with the DELPHI detector at LEP. Astropart. Phys. 2007, 28, 273-286. [CrossRef]

42. Watson, A.A. Conclusions about properties of high-energy cosmic-rays drawn with limited recourse to hadronic models. Nucl. Part. Phys. Proc. 2017, 291-293, 66-73. [CrossRef]

43. Thomas, K. Gaisser, Spectrum of cosmic-ray nucleons, kaon production, and the atmospheric muon charge ratio. Astrop. Phys. 2012, 35, 801-806.

44. Rebel, H.; Sima, O. What could we learn from observations of the muon charge ratio in cosmic ray air showers? Rom. Rep. Phys. 2007, 59, 609-624.

45. CMS Collaboration. Measurement of the charge ratio of atmospheric muons with the CMS detector. Phys. Lett. B 2010, 692, 83-104.

46. MINOS Collaboration. Measurement of the multiple-muon charge ratio in the MINOS Far Detector. Phys. Rev. D 2016, 93, 052017. [CrossRef]

47. Ostavchenko, S. Monte Carlo treatment of hadronic interactions in enhanced Pomeron scheme: I. QGSJET-II model. Phys. Rev. D 2011, 83, 014018. [CrossRef]

48. The TOTEM Collaboration. First measurement of the total proton-proton cross section at the LHC energy of $\sqrt{s}=7$ TeV. Europhys. Lett. 2011, 96, 21002. [CrossRef]

49. ALICE Collaboration. Study of cosmic ray events with high muon multiplicity using the ALICE detector at the CERN Large Hadron Collider. JCAP 2016, 1, 32.

50. Heck, D.; Knapp, J.; Capdevielle, J.N.; Schatz, G.; Thouw, T. CORSIKA: A Monte Carlo Code to Simulate Extensive Air Showers. FZKA-6019. 1998. Available online: https://web.ikp.kit.edu/corsika/physics_ description/corsika_phys.pdf (accessed on 13 October 2020).

51. Kankiewicz, P.; Rybczynski M.; Wlodarczyk1 Z.; Wilk G. Muon Bundles as a Sign of Strangelets from the Universe. Astrophys. J. 2017, 839, 31. [CrossRef]

52. Ridky, J. Can we observe the Quark Gluon Plasma in Cosmic Ray showers? Astropart. Phys. 2002, 17, 355-365. [CrossRef]

53. ATLAS Collaboration. The ATLAS Experiment at the CERN Large Hadron Collider. Jinst 2008, 3, S08003.

54. Curtin D.; Drewes, M.; McCullough, M.; Meade, P.; Mohapatra, R.N.; Shelton, J.; Shuve, B.; Accomando, E.; Alpigiani, C.; Antusch, C.; et al. Long-Lived Particles at the Energy Frontier: The MATHUSLA Physics Case. Rept. Prog. Phys. 2019, 82, 116201. [CrossRef]

55. Salam, A.; Strathdee, J. Super-symmetry and non-Abelian gauges. Phys. Lett. B 1974, 51, 353-355. [CrossRef]

56. Mathusla Collaboration. An Update to the Letter of Intent for MATHUSLA: Search for Long-Lived Particles at the HL-LHC. arXiv 2020, arXiv:2009.01693.

57. Stanev, T. High Energy Cosmic Rays, 2nd ed.; Springer: New York, NY, USA, 2004.

58. Greisen, K. Progress in Cosmic Ray Physics; JG Wilson: Amsterdam, The Netherlands, 1958.

59. Tapia, A.; Melo, D.; Sánchez, F.; Sedoski Croce, A.; Etchegoyen, A.; Figueira, J.M.; Gamarra, R.F.; García, B.; González, N.; Josebachuili, M.; et al. The lateral shower age parameter as an estimator of chemical composition. arXiv 2013, arXiv:1309.3536.

60. ARGO-YBJ collaboration. EAS age determination from the study of the lateral distribution of charged particles near the shower axis with the ARGO-YBJ experiment. Astrop. Phys. 2017, 93, 46-55. [CrossRef]

61. ARGO Collaboration. Layout and performance of the RPCs used in the ARGO-YBJ experiment. Nucl. Instr. Meth. A 2006, 562, 92-96. [CrossRef]

62. KASCADE Collaboration. Measurements of Attenuation and Absorption Lengths with the KASCADE Experiment. Astrop. Phys. 2003, 19, 703-714. [CrossRef]

63. Joshi, V.; Jardin-Blicq, A.; The HAWC Collaboration. HAWC High Energy Upgrade with a Sparse Outrigger Array. In Proceedings of the 35th International Cosmic Ray Conference, Busan, Korea, 10-20 July 2017. 
64. TAIGA Collaboration. TAIGA-HiSCORE: Results from the first two operation seasons. In Proceedings of the 35th International Cosmic Ray Conference, Busan, Korea, 10-20 July 2017.

65. The IceCube-Gen2 Collaboration. The IceTop Scintillator Upgrade. In Proceedings of the 35th International Cosmic Ray Conference, Busan, Korea, 10-20 July 2017.

66. Tareq AbuZayyad for the Telescope Array Collaboration. Cosmic Rays Energy Spectrum from PeV to EeV energies measured by the TALE detector. In Proceedings of the 35th International Cosmic Ray Conference, Busan, Korea, 10-20 July 2017.

67. Di Sciascio, G. The LHAASO experiment: from Gamma-Ray Astronomy to Cosmic Rays. Nucl. Phys. Proc. 2019, 10, 1-8. [CrossRef]

68. Sokolsky, P. Introduction to Ultrahigh Energy Cosmic Ray Physics; Addison-Wesley Publishing Company: Boston, MA, USA, 1989.

69. KASCADE Collaboration. KASCADE measurements of energy spectra for elemental groups of cosmic rays: Results and open problems. Astrop. Phys. 2005, 24, 1-25. [CrossRef]

Publisher's Note: MDPI stays neutral with regard to jurisdictional claims in published maps and institutional affiliations.

(C) 2020 by the authors. Licensee MDPI, Basel, Switzerland. This article is an open access article distributed under the terms and conditions of the Creative Commons Attribution (CC BY) license (http:/ / creativecommons.org/licenses/by/4.0/). 